\title{
Search for fullerenes and PAHs in the diffuse interstellar medium
}

\author{
P. Ehrenfreund' and B. H. Foing ${ }^{2}$ \\ 'Leiden Observatory, P.O. Box 9513, NL-2300 RA Leiden, The Netherlands \\ ${ }^{2}$ Solar System Division, ESA/SSD, ESTEC/SO, 2200 Noordwijk AG, The Netherlands
}

Received 26 October 1994; revised and accepted 30 January 1995

\begin{abstract}
Recent studies suggest carbon-containing molecules as the best candidates for carriers of the unidentified diffuse interstellar bands (DIBs), considering their abundance and ability to form stable bonds in interstellar space. We have searched for new DIBs in the near-IR and have detected two new diffuse bands that are consistent with laboratory measurements of $\mathrm{C}_{60}^{+}$in a neon matrix. Criteria for this possible identification are discussed. From these observations and the DIB measured absorption, we estimate that up to $0.9 \%$ of interstellar carbon could be in the form of $\mathrm{C}_{60}^{+}$. We also searched for polycyclic aromatic hydrocarbon $(\mathrm{PAH})$ cations and have derived corresponding limits for the presence of the coronene $\mathrm{C}_{24} \mathrm{H}_{12}$ and ovalene $\mathrm{C}_{32} \mathrm{H}_{34}$ cations in space. We have studied the ionization properties of these PAH cations, which could explain their selective destruction. From these results we discuss the role of fullerenes and PAHs as possible DIB carriers.
\end{abstract}

\section{Diffuse interstellar bands, polycyclic aromatic hydrocarbons and fullerenes}

Astronomical observations to date have revealed 150 diffuse interstellar bands (DIBs) at wavelengths ranging from the blue part of the visible spectrum to the near-IR (Herbig, 1975; Herbig and Leka, 1991; Jenniskens and Desert, 1994).

Polycyclic aromatic hydrocarbons (PAHs), fullerenes and their ions were suggested to be interesting carrier molecules for the DIBs (Leger and d'Hendecourt, 1985 ; van der Zwet and Allamandola, 1985 ; Kroto, 1987 ; Leger et al., 1988; Kroto and Jura, 1992). The fullerene $\mathrm{C}_{60}$ has subsequently attracted much attention since it was synthesized by vaporizing graphite in a helium atmosphere (Kraetschmer et al., 1990). $\mathrm{C}_{60}$ is expected to be

Correspondence to : P. Ehrenfreund the most stable and dominant fullerene during carbon clustering. The ability of hydrogen to inhibit the fullerene growth mechanism will limit the formation of $\mathrm{C}_{60}$ to very specific hydrogen-depleted environments in space, but once formed, $\mathrm{C}_{60}$ may be stable and survive long enough to be cycled into the diffuse medium. The low ionization potential of $\mathrm{C}_{60}(7.61 \mathrm{eV})$ will also favour its ionization in the diffuse medium outside of dark clouds.

PAHs, present as a mixture of neutral and ionized species, are likely responsible for the set of IR emission bands in the 2-1 $5 \mu \mathrm{m}$ range, which are observed in many different objects such as reflection and planetary nebulae, H II regions and even external galaxies (Leger and Puget, 1984). PAHs are suggested to be the most abundant free organic molecules, ubiquitous in space and remarkably stable (Puget and Léger, 1989). The suggestion that a mixture of different PAHs and fullerene compounds could explain some of the DIBs therefore seems plausible. Observations of DIBs in dense environments also indicate an influence of photoionizing conditions (Jenniskens et al., 1994; Ehrenfreund and Jenniskens, 1995).

Laboratory measurements, using rare gas matrix isolation techniques and UV photolysis, have recently provided a set of spectra of PAHs, PAH ions and fullerenes. These data allow a good comparison with interstellar data or even a search for specific carrier molecules in the interstellar medium. Spectra of the naphthalene and pyrene cations in a solid neon matrix were reported recently, indicating a link between these small PAH ions and the diffuse bands (Salama and Allamandola, 199 1, 1992a, b). The spectra of the coronene and ovalene cations have been measured in solid neon (Ehrenfreund et al., 1992, 1994) and recently the spectrum of $\mathrm{C}_{60}^{+}$has been measured in both argon and neon matrices (Kato et al., 1991; Gasyna et al., 1992 ; d'Hendecourt et al,, 1992 ; Fulara et al., 1993).

$\mathrm{C}_{60}^{+}$and the coronene and ovalene cations all show strong transitions in the near-IR. Whereas diffuse interstellar features have been extensively studied between 4400 and $8600 \AA$, astronomical measurements below $4400 \AA$ and in the near-IR are scarce, and only a few diffuse bands 
have been detected in those ranges (Hibbins et al., 1994; Joblin et al., 1990; Foing and Ehrenfreund, 1994, 1995).

We have searched for new DIBs in the near-IR, specifically to identify $\mathrm{C}_{60}^{+}$and the coronene and ovalene cations in the diffuse medium. Due to their pericondensed structure, coronene and ovalene are believed to be among the most stable PAHs in the interstellar medium.

Observations were performed with the $1.52 \mathrm{~m}$ Coude telescope/spectrograph Aurelie at Observatoire de Haute Provence (OHP), France with a spectral resolving power $R=36.000$ or $\Delta \lambda=0.27 \AA$. The spectral domain at 9500 $\AA$ is significantly absorbed by the Earth's atmospheric water and requires a detailed telluric correction.

\section{$\mathrm{C}_{60}^{+}$in the diffuse interstellar medium}

The spectrum of $\mathrm{C}_{60}^{+}$in rare gas matrices shows two dominant peaks at $9663+9$ and $9724+4 \AA$ in argon and at $9580 \pm 4$ and $9642 \pm 3 \AA$ in neon (Gasyna et al., 1992; d'Hendecourt et al., 1992 ; Fulara et al., 1993). These bands are five times stronger than any other features in the $5000-10,000 \AA$ range.

We found two diffuse bands satisfying the classical criteria for DIBs in the near-IR at 9577 and $9632 \AA$ that are coincident (within $0.1 \%$ ) with laboratory measurements of $\mathrm{C}_{60}^{+}$in a neon matrix. Figure 1 shows a sequence of
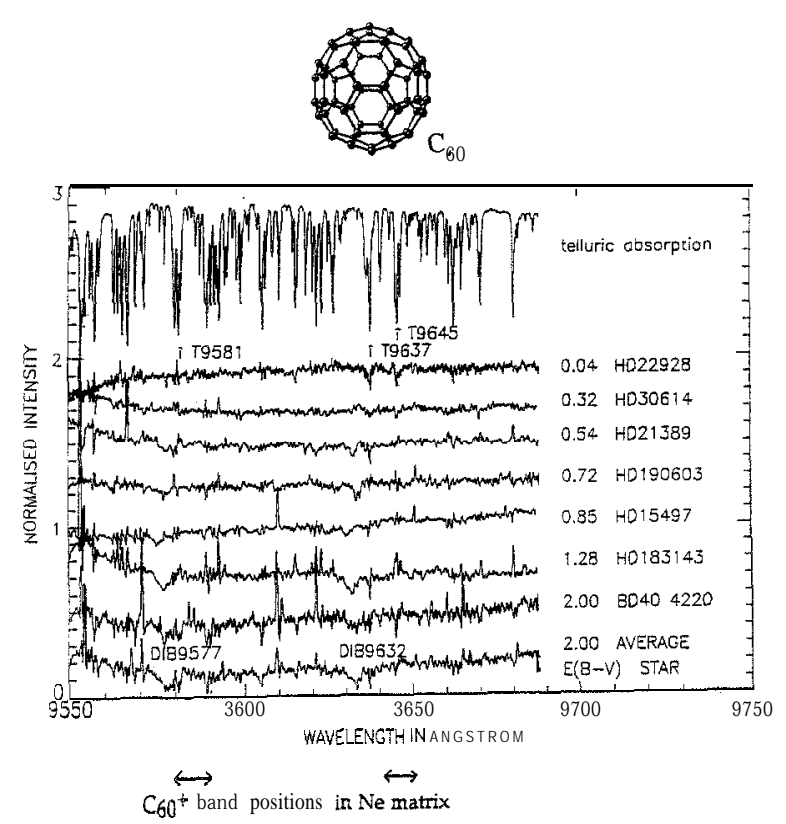

Fig. 1. Sequence of reduced spectra (normalized to unity) of program stars with increasing reddening $E_{(B-\eta)}$, centred at 9650 $\AA$. Each observed stellar spectrum has been divided after instrumental corrections by a spectrum of a reference star of similar spectral type. The top trace is a reference spectrum of $\eta$ Ursae Majoris showing the strength of the telluric bands. Slight residuals from telluric corrections are indicated (T). Two new DIBs are detected at 9577 and $9632 \AA$, increasing with $E_{(B-V)}$ The slight wavelength shifts up to $1.3 \AA$ and different line profiles are partly associated with the velocity distribution of interstellar clouds along the line of sight and possibly reflect intrinsic changes in the line broadening. An average composite spectrum corresponding to $E_{\left(B \cdot \eta^{n}\right)}=2$ is shown at the bottom. We also give the position of the two $\mathrm{C}_{60}^{+}$main bands measured in a neon matrix by Fulara et al. (1993) and d'Hendecourt et al. (1992) reduced spectra (normalized to unity) of program stars with increasing reddening $E_{(B-\eta)}$ (Foing and Ehrenfreund, 1994, 1995).

\section{Criteria for the spectroscopic identification of $\mathrm{C}_{60}^{+}$}

Several criteria were addressed to ensure a consistent identification of a DIB carrier with the specific candidate $\mathrm{C}_{60}^{+}$(Foing and Ehrenfreund, 1994, 1995). An important parameter to know is the shift in position of the absorption peak due to the neon or argon matrices, compared to the gas phase. From a comparison of gas phase and neon matrix spectra, a shift of $50 \mathrm{~cm}^{-1}$ has been reported at $6300 \AA$ for the lowest electronic transitions of neutral $C_{60}$ (Haufler et al., 1991 ; Maier, 1994). In fact, from the same measurements we derive that the shift decreases systematically when going to the red. Is the shift comparable or larger/smaller for $\mathrm{C}_{60}^{+}$transitions around 9600 A?

According to Bondybey and Miller (1983), smaller shifts between gas phase and neon matrix data are expected for IR transitions involving deeper states, whereas excited states are more strongly affected and lead to larger shifts. Therefore, a shift of only $10 \mathrm{~cm}^{-1}$ for the IR transition of $\mathrm{C}_{60}^{+}$remains plausible.

It has been argued that the two bands appearing in the neon matrix might be due to a matrix splitting effect. Because the two cation bands appear in argon and neon matrices with the same average separation $(61 \pm 10,62 \pm 6$ A), they are probably intrinsic to two major ground state geometries favoured by Jahn-Teller types of distortion and not a matrix splitting effect due to different kinds of sites.

According to Bendale et al. (1992), the ground state of $\mathrm{C}_{60}^{-}$departs from the $I_{h}$ optimized symmetry of neutral $\mathrm{C}_{60}$ (two transitions $H_{g} \rightarrow H_{u}$ and $G_{g} \rightarrow H_{u}$, well separated by $300 \mathrm{~cm}^{-1}$ ) towards the $D_{5 d}$ geometry (five degenerate transitions $E_{1 g} \rightarrow A_{1 u}$ ), which is the preferred static equilibrium structure with a Jahn-Teller distortion stabilization energy of $8.1 \mathrm{kcal} \mathrm{mol}^{-1}$. These theoretical calculations are still consistent with the two measured transitions, separated by only $56 \mathrm{~cm}^{-}$.

According to Maier (1994), the two corresponding peaks in the neon matrix spectrum vary in ratio from $1: 1.5$ to $1: 2$, depending on the production conditions, and are attributed to the two distorted forms of $\mathrm{C}_{60}^{+}(\mathrm{Ful}-$ ara et al., 1993). The spectrum of $\mathrm{C}_{60}^{+}$in a neon matrix measured by d'Hendecourt et al. (1992) also shows an intensity ratio of $1: 1.5$ for the two dominant bands, as does the published spectrum of $\mathrm{C}_{60}^{+}$measured by Fulara et al. (1993).

The observed relative strength of the two new DIBs is $1: 1.6$, with an error of $30 \%$ due to residual telluric contaminations in observed spectra. This consistency is another piece of evidence for our identification of these bands.

The results presented show strong evidence for the existence of the $\mathrm{C}_{60}$ cation as the largest molecule ever detected in space, and likely provide the first direct identification of diffuse interstellar bands (Foing and Ehrenfreund, 1994). 


\section{$\mathrm{C}_{60}^{+}$abundance}

Assuming an oscillator strength for $\mathrm{C}_{60}^{+}$of 0.1 according to theoretical estimates (Bendale et al., 1992), we calculate [after Spitzer (1978)] from A $W_{\lambda}=\lambda^{2} N f \pi e^{2} / m c^{2}$, assuming a ratio $\mathrm{C} / \mathrm{H}=3.7 \times 10^{-4}$ and hydrogen column density per unit interstellar absorption $N_{\mathrm{H}} / A_{v}=2 \times 10^{21} \mathrm{~cm}^{-2}$, with $A_{v}=3 E_{(B-V)}$, that $0.035 \%$ of the cosmic carbon could be in the form of $\mathrm{C}_{60}^{+}$Taking the lower oscillator strength limit of 0.004-0.012 derived from the experiments of Kato et al. (1991), which is probably the more accurate estimate, this becomes a maximum of $0.3-0.9 \%$ of the cosmic carbon in $\mathrm{C}_{60}^{+}$. This indicates that $\mathrm{C}_{60}^{+}$may play an important role in interstellar chemistry.

At least two more bands due to the vibrational excitation in the upper electronic state should be apparent with $15 \%$ of the strength of the $957719632 \AA$ bands in the near-IR around 9421 and $9366 \AA$. It will be difficult to observe these weak bands due to strong telluric and stellar contaminations in this wavelength range. However, these lines should be searched for in interstellar space from very dry observing sites to confirm our results.

Future work should also include the gas phase spectrum of $\mathrm{C}_{60}^{+}$, more astronomical observations to study $\mathrm{C}_{60}^{+}$in different environments, the search for neutral $\mathrm{C}_{60}$ and the study of other fullerene compounds-exohedral and endohedral metallo-complexes and fulleranes. A search for other fullerene compounds is already underway.

\section{Coronene cation abundance in tbe diffuse medium}

The electronic absorption spectrum of the coronene cation obtained in a neon matrix shows two strong absorption bands at $4592 \AA\left(f=1.2 \times 10^{-2}\right.$ and $\left.\mathrm{FWHM}=28 \AA\right)$ and $9465 \AA\left(f=1.8 \times 10^{-3}\right.$ and FWHM $\left.=16 \AA\right)$ (Ehrenfreund et al., 1992).

The band at $9465 \AA$ corresponds to the first ionic transition of coronene, from orbital $E_{2 u}$ to $E_{1 g}$, is symmetryallowed and shows a strong vibronic progression towards shorter wavelengths. The wavelength region around 9465 $\AA$ is very difficult to observe due to strong telluric absorption. In telluric-corrected spectra, a band at $9466 \AA$ is found, located at the exact wavelength of the laboratory transition (Ehrenfreund et al.,1995). However, the band is weak and narrow (FWHM $=2 \AA$ ) and could be partly masked by a stellar transition such as Na I (9465.9 A).

Coronene has an additional strong, well-defined transition at $4592 \AA$ (measured in a neon matrix), which can be used for identification. Figure 2 shows a series of spectra of stars in this range divided by standards of the same or very similar spectral type. A narrow feature at $4583 \AA$ is observed in two obscured stars but coincides with a number of stellar lines $(4582.98 \AA$ for Ne I, $4583.44 \AA$ for Ti II, $4583.84 \AA$ for Fe II). The feature may thus be a remnant of imperfect stellar line subtraction rather than a DIB. Note that near $4550 \AA$ a similar feature is found, as are a number of stellar lines that normally obliterate weak DIBs (4549.19 and $4549.47 \AA$ for Fe II. $4549.62 \AA$ for Ti II, $4550.64 \AA$ for Ne I). However, the absence of both bands in HD 217086 suggests that these are stellar lines (most

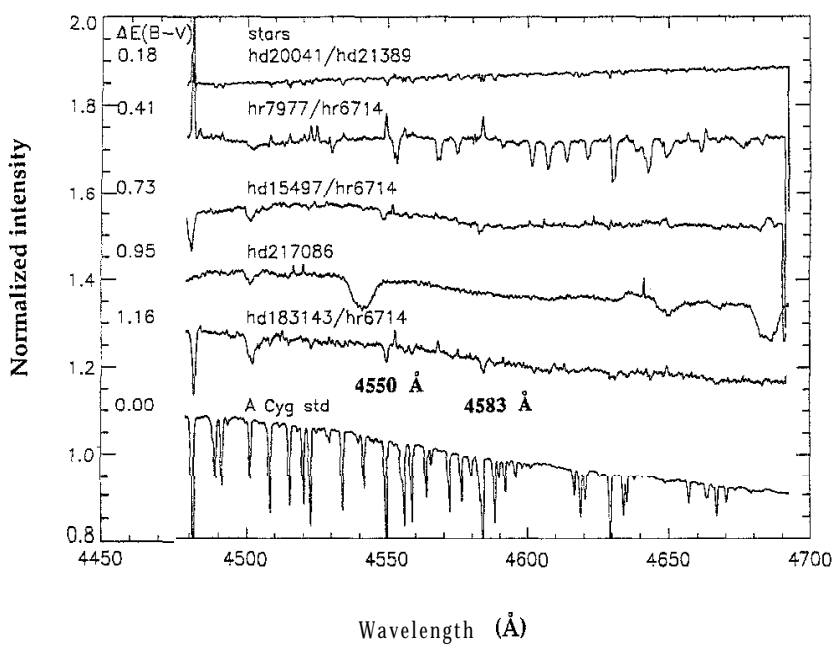

Fig. 2. Sequence of reduced spectra of program stars (normalized to unity and offset) with increasing reddening $E_{(B \cdot \hbar)}$, centred at $4580 \AA$ A Each observed stellar spectrum has been divided after instrumental corrections by a spectrum of a reference star of similar spectral type and the difference of reddening $\Delta E_{(B-V)}$ is also indicated. The known DIB at $4501.8 \AA$ can be seen in all spectra. Narrow features ascribed to residuals of stellar line corrections were found at 4550 and $4583 \AA$ in the obscured stars HD15497 and HD183143. A shallow feature at $4600 \AA$ (FWHM $=40 \AA$ and optical depth of 0.04 ) appears in the most reddened stars, but with a varying shape, position and strength, which is not consistent with a classical DIB

probably Fe II). Therefore, no new narrow DIBs above $70 \mathrm{~m} \AA / E_{(B-V)}$ are found in this wavelength range.

A broad band at $4600 \AA$ (FWHM $=30 \mathrm{~A})$, observed in several stars, is listed in Jenniskens and Desert (1994) as a doubtful DIB with an average equivalent width of $450 \mathrm{~m} \AA$ normalized to $E_{(B-\eta)}=1$. This shallow feature also appears in our spectra and seems real, but is difficult to associate with a unique classical interstellar DIB, because its position and shape vary in the three more reddened stars and its strength does not increase with reddening.

Based on the observational limits and the known oscillator strength for the coronene cation bands (Ehrenfreund et al., 1992), we estimate an upper limit for the abundance. Using the total equivalent width of $70 \mathrm{~m} \AA$ (4592 $\AA$ band) per unit reddening $E_{(B-\vartheta)}$ and $75 \mathrm{~m} \AA$ (9466 $\AA$ band) derived from our observations, we calculate that less than $0.05 \%$ of the cosmic carbon could be in the form of the coronene cation.

\section{Upper limit on the ovalene cation abundance}

The second transition of the ovalene ion from orbital $B_{2 g}$ to $A_{u}$ has recently been measured by d'Hendecourt (Ehrenfreund et al., 1995) in a neon matrix. This band appears at $9780 \AA$ and is symmetry allowed.

Figure 3 shows a series of the telluric-corrected spectra centred at $9780 \AA$ in order to identify this transition of the ovalene cation. A composite spectrum combining the four reddened stars between 0.54 and 1.28 and scaled to 


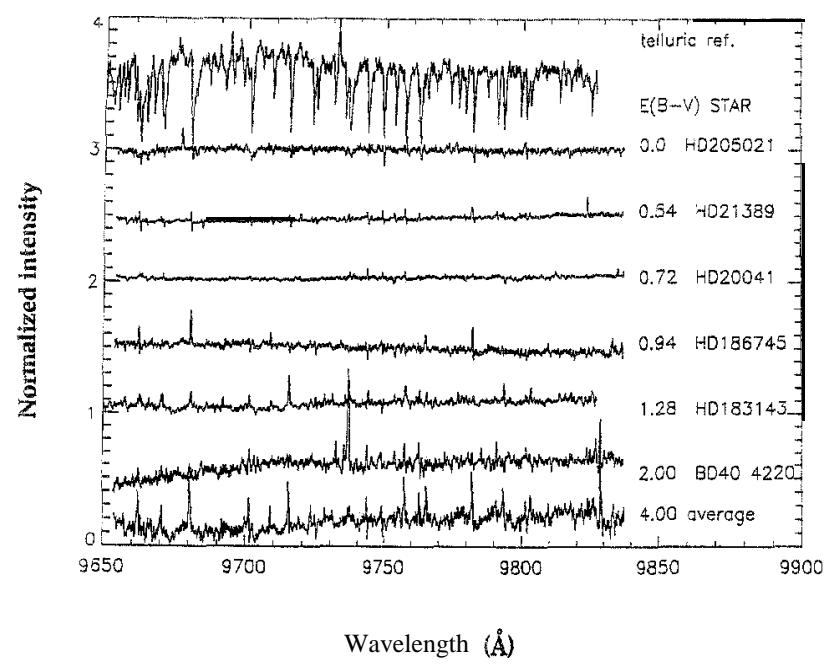

Fig. 3. Sequence of reduced spectra (normalized to unity) of program stars with increasing reddening $E_{(B-V)}$, centred at 9750 A. Each observed stellar spectrum has been divided after instrumental corrections by a spectrum of a reference star of similar spectral type. No DIBs stronger than $30 \mathrm{~m} \AA$ per unit reddening were observed in this range. An average composite spectrum corresponding to $E_{(B-V)}=4$ is shown at the bottom, which displays small features at 9672,9783 and $9798 \AA$ (Foing and Ehrenfreund, 1995)

$E_{(B-\curvearrowleft)}=4$ is also shown in Fig. 3. This region is relatively free from telluric remnants, which appear as isolated spikes in the spectra, and surprisingly displays no strong DIBs. We find a possible, though weak, counterpart in the astronomical spectrum at $9783 \AA$ with a measured equivalent width of $30 \mathrm{~m} \AA$ per unit reddening. There are no further bands stronger than $30 \mathrm{~m} \AA / E_{(B-V)}$ observed within $\pm 100 \mathrm{~A}$.

The second transition of the ovalene cation appears very strong in a neon matrix, and therefore a high oscillator strength $(f)$ is possible up to 0.01 , in analogy with the second transition of the coronene ion at $4592 \AA$ $\left(f=1.2 \times 10^{-2}\right)$. If the band at $9783 \AA$ is attributed to the ovalene cation, the abundance of ovalene is very low (as has been observed for the coronene cation).

Assuming $0.001<f<0.01$ and a DIB with $30 \mathrm{~m} \AA /$ $E_{(B-Y)}$, the amount of cosmic carbon locked up in the ovalene ion would range from 0.05 to $0.005 \%$. The oscillator strength of this transition is currently being determined in the laboratory.

\section{Photoionization of PAH and fullerene cations}

On the basis of laboratory measurements of the coronene and ovalene cations, and by comparison with astronomical spectra at $4590 \AA$ and in the near-IR (at 9465 and 9780 A) in different environments, weak features could be detected above the noise level, leading to an abundance limit determination for these molecules, We calculate from our results that coronene and ovalene cations, if present, could account for as much as $0.05 \%$ of the cosmic carbon. These abundances are typically smaller than those of naphthalene and pyrene (or their substituted homologues), reported to account for $0.2-0.3 \%$ of the cosmic carbon by Salama and Allamandola (1993). On the other hand, $\mathrm{C}_{60}^{+}$could represent up to $0.9 \%$ of the cosmic carbon. How can the different abundances be explained?

Considering PAH ions as possible DIB carriers, we should compare how their ionization properties and destruction pathways relate to current observations. Destruction of PAHs probably involves doubly charged PAHs (Leach, 1987). Jochims et al. (1994) argue that nascent PAHs ions of any size formed by photoionization in HI regions will preferentially relax by IR emission. Those that have $N_{c}<3040$ will tend to relax by photofragmentation when doubly ionized, rather than by IR relaxation.

The double ionization potential of 28 PAHs of different size and structures have recently been measured by Tobita et al. (1994). These data show that small PAHs (up to 14 carbon atoms) have, in general, an ionization potential from cation to dication (AI) above $12 \mathrm{eV}$ and the majority above $13 \mathrm{eV}$. Small molecules like naphthalene therefore require higher energy photons to be doubly ionized.

Generally, a AI smaller than $12 \mathrm{eV}$ is seen for molecules containing more than 18-20 carbon atoms. Certainly, the molecular structure also plays a role (Ehrenfreund et al., 1995). Up to now, a limited set of astronomical observations shows that small molecules with a somewhat higher ionization energy from $\mathrm{PAH}^{+}$to $\mathrm{PAH}^{2+}$ (AI), like naphthalene $(A I=13.35 \mathrm{eV})$ and pyrene $(\mathrm{AI}=12.35 \mathrm{eV})$, show coincidences in band positions with observed features in the interstellar medium. Molecules of intermediate size like coronene (with lower $\Delta I=11.5 \mathrm{eV}$ ) and ovalene $(\mathrm{AI}=11.3 \mathrm{eV})$ do not have obvious counterparts in astronomical spectra.

Looking at the interstellar radiation field, we know that near the ionization potential of hydrogen at $13.6 \mathrm{eV}$, very few photons are available due to the efficient absorption by the $\mathrm{H}$ Lyman continuum above $13.6 \mathrm{eV}$, by molecules like $\mathrm{CO}$ and $\mathrm{H}_{2}$ just below, and increased dust extinction at shorter wavelengths.

The ionization rate $\eta=\int_{\Delta I} \sigma_{v} \varphi_{v} \mathrm{~d} E$, where $\sigma_{v}$ is the cross-section and $\varphi_{\nu}$ is the UV field, varies drastically with the ionization potential AI. Taking a Draine (1978) field for $\varphi_{y}=1.658 \times 10^{6} E-2.152 \times 10^{5} E^{2}+6.919 \times 10^{3} E^{3}$ in units of photon $\mathrm{cm}^{-2} \mathrm{~s}^{-1} \mathrm{sr}^{-1} \mathrm{eV}^{-1}$ with $E$ in $\mathrm{eV}$ and taking a cross-section $\sigma_{v}=\sigma_{0}=10^{-16} \mathrm{~cm}$ ' (Leach, 1987), we find a photoionization rate of $1.7 \times 10^{-9} \mathrm{~s}^{-1}$ for the coronene ion $(\mathrm{A} 1=11.5 \mathrm{eV})$, six times faster than the $1 \times 10^{-10} \mathrm{~s}^{-1}$ found for the naphthalene ion $(\Delta I=13.35$ $\mathrm{eV})$. The corresponding rates can be compared for PAHs with different second ionization energies (Fig. 4), given the UV field (Draine, 1978) and the integrated UV field between the ionization energy and the $13.6 \mathrm{eV}$ Lyman cut-off.

Due to the strong gradient in the short wavelength dust extinction, the UV field slope will be dramatically modified in obscured regions. This will shield small molecules like naphthalene and pyrene cations with a $A I$ near $13 \mathrm{eV}$ from being doubly ionized.

It has to be stated that PAH cations which are possible DIB carriers are just a part of the interstellar medium inventory of molecules. PAHs responsible for the IR emission may be of much larger size and may not be readily destroyed by such a double ionization process.

However, for the diffuse medium with normal UV con- 


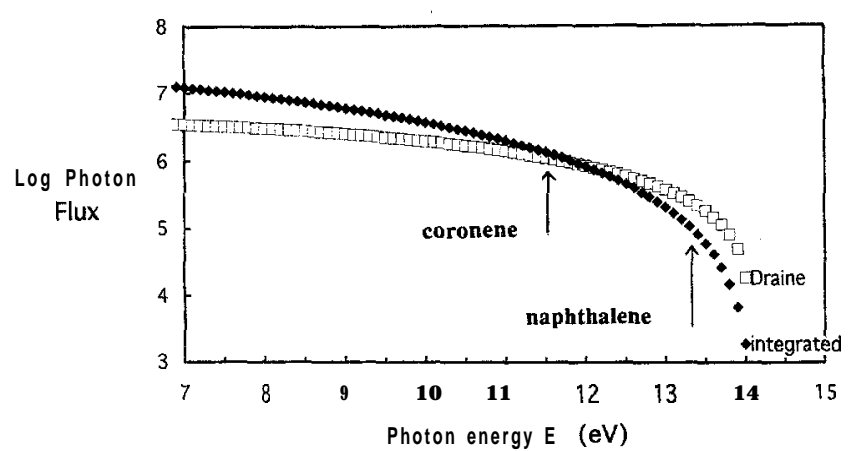

Fig. 4. Dependence on ionization rates of various PAHs according to the UV interstellar field. The UV field (Draine, 1978) $\varphi$ in units of photon $\mathrm{cm}^{-2} \mathrm{~s}^{-1} \mathrm{sr}^{-1} \mathrm{eV}^{-1}$ and the integrated UV field $I_{\mathrm{UV}}$ beyond the cation ionization potential IP (in photons $\mathrm{cm}^{-2} \mathrm{sr}^{-1}$ ) are given. The ionization potentials (IP) of some typical PAH cations are indicated. The ionization rates can be estimated by multiplying $I_{\mathrm{UV}}$ by $4 \pi \sigma$, where $\sigma$ is the PAH ionization cross-section

ditions, our results imply that the survival of PAHs (of up to 40 carbon atoms) may be determined by the photoionization of the cation near the $13.6 \mathrm{eV}$ cut-off, leading to dications that would subsequently be fragmented. Similar $\mathrm{UV}$ conditions will ionize both $\mathrm{C}_{60}^{+}$(second ionization potential $11.3 \mathrm{eV}$ ) and PAHs, but the corresponding $\mathrm{C}_{60}$ dication is very stable against fragmentation (thanks to the high dissociation barrier of the fullerene cage structure) and can revert to the monocation through electronic recombination. This would explain the significant equilibrium abundance of $\mathrm{C}_{60}^{+}$observed in the diffuse interstellar medium.

Acknowledgements. We thank Louis d'Hendecourt. Peter Jenniskens and Xavier Désert for their contributions, and Tom Millar and Werner Schmidt for extended and fruitful discussions. We also thank A. Collier-Cameron and the staff of OHP for their efficient support during the observations. P. E. is a recipient of a fellowship of the European Community, ERBCHBICT940939.

\section{References}

Bendale, R. D., Stanton, J. F. and Zerner, M. C., Chem. Phys. Lett. 194,467, 1992.

Bondybey, V. E. and Miller, T. A., Molecular Ion Spectroscopy, Structure, and Chemistry (edited by T. Miller and V. E. Bondybey), p. 125. North Holland, Amsterdam, 1983.

Draine, B. T., Astrophys. J. Suppl. 36, 595, 1978.

Ehrenfrennd, P. and Jenniskens, P., in Diffuse Znterstellar Bands (edited by A. Tielens and T. Snow). Kluwer, Dordrecht, 1995, in press

Ehrenfreund, P., d’Hendecourt, L., Verstraete, L., Léger, A., Schmidt, W. and Defourneau, D., Astron. Astrophys. 295,257, 1992.

Ehrenfreund, P., Foing, B., d'Hendecourt, L., Jenniskens, P. and Désert, X., Astron. Astrophys. 1995, in press.

Foing, B. H. and Ehrenfreund, P., Nature 369, 296, 1994.

Foing, B. H. and Ehrenfreund, P., in Diffuse Znterstellar Bands (edited by A. Tielens and T. Snow). Kluwer, Dordrecht, 1995, in press.

Fulara, J., Jakobi, M. and Maier, J. P., Chem. Phys. Lett. 211, 227, 1993.

Gasyna, Z., Andrews, L. and Schatz, P. N., J.phys. Chem. 96 , $1525,1992$.

Haufler, R. et al., J. Chem. Phys. 95, 2197, 1991.

d'Hendecourt, L. et al., in C. Joblin thesis, Université Paris VII, 1992.

Herbig, G. H., Astrophys. J. 196, 129, 1975.

Herbig, G. H. and Leka, K. D., Astrophys. J. 382, 193, 1991.

Hibbins et al., Proceedings "Diffuse Znterstellar Bands", Contributed Papers, 31, 1994.

Jenniskens, P. and Désert, X., Astron. Astrophys. 106, 39, 1994.

Jenniskens, P., Ehrenfreund, P. and Foing, B. H., Astron. Astrophys. 281,517, 1994.

Joblin, C., Maillard, J. P., d'Hendecourt, L. and Léger, A, Nature 346, 729, 1990.

Jochims, H. W., Ruehl, E., Baumgaertel, H., Tobita, S. and Leach, S., Astrophys. J.420, 307, 1994.

Kato, T. et al., Phys. Lett. 180,446, 1991.

Kraetschmer, W., Lamb, L., Fostiropoulos, K. and Huffman, D. R., Nature 347, 354, 1990.

Kroto, H. W., in Polycyclic Aromatic Hydrocarbons and Astrophysics (edited by A. Léger and L. d'Hendecourt), p. 197. Reidel, Dordrecht, 1987.

Kroto, H. W. and Jura, M., Astron. Astrophys. 263, 275-280, 1992.

Leach, S., in Polycyclic Aromatic Hydrocarbons and Astrophysics (edited by A. Léger and L. d'Hendecourt), pp. 99-129. Reidel, Dordrecht; 1987.

Léger, A. and d'Hendecourt, L., Astron. Astrophys. 146, 81, 1985.

Léger, A. and Puget, J. L., Astron. Astrophys. 137, L5, 1984.

Léger, A., d'Hendecourt, L., Verstraete, L. and Schmidt, W., Astron. Astrophys. 203,415, 1988.

Maier, J. P., Nature 370,423, 1994.

Puget, J. L. and Léger, A., Astron. Astrophys. Rev. 27, 161, 1989.

Salama, F. and Allamandola, L., J. Chem. Phys. 94, 6964, 1991.

Salama, F. and Allamandola, L., Astrophys. J. 395, 301-306, $1992 a$.

Salama, F. and Allamandola, L., Nature 358, 42-43, 1992 b.

Salama, F. and Allamandola, L., J. chem. Soc., Faraday Trans. 89, 2277, 1993.

Spitzer, L., in Physical Processes in the Znterstellar Medium. Wiley, New York, 1978.

Tobita, S., Leach, S., Jochims, H., Rühl, E., Illenberger, E. and Baumgärtel, H., Can. J. Phys. 72, 1060-1069, 1994.

van der Zwet, G. P. and Allamandola, L. J., Astron. Astrophys. 146, 76, 1985. 